\title{
Migräne erhöht das kardiovaskuläre Risiko
}

\author{
Bei Frauen mit Migräne ist das Risiko für schwerwiegende kardiovaskuläre Erkrankungen, Herz- \\ infarkt, Schlaganfall und die Notwendigkeit von koronaren Revaskularisationen leicht erhöht.
}

Frauen, die unter einer Migräne mit Aura leiden, tragen ein leicht erhöhtes Schlaganfallrisiko. Für andere kardiovaskuläre Erkrankungen gab es bisher wenige Daten. Diese Lücke schließt nun eine Auswertung der Nurses' Health Study, für die ab 1989 in den USA 116.430 Krankenschwestern im Alter von 25-42 Jahren ohne vaskuläre Erkrankungen rekrutiert wurden. Die letzten Verlaufsuntersuchungen erfolgten 2011.

Um den Zusammenhang zwischen Migräne und kardiovaskulären Erkrankungen zu ergründen, wurden die Daten von 115.541 Teilnehmerinnen ausgewertet. Bei Befragungen in den Jahren 1989, 1993 und 1995 hatten sie berichtet, ob bei ihnen Migräne diagnostiziert worden war. Vaskuläre Ereignisse wurden alle zwei Jahre per Fragebogen erfasst.
17.531 Frauen $(15,2 \%)$ berichteten, unter einer Migräne zu leiden. Während der 20-jährigen Beobachtungszeit traten 1.329 schwerwiegende kardiovaskuläre Ereignisse auf. 223 Frauen verstarben an kardiovaskulären Erkrankungen.

Die Migräne war signifikant mit schwerwiegenden kardiovaskulären Erkrankungen (Hazard Ratio [HR] 1,50), Myokardinfarkt (HR 1,39), Schlaganfall (HR 1,62) sowie mit Angina pectoris und koronaren Revaskularisationen (HR 1,73) assoziiert. Die Zusammenhänge waren unabhängig von Alter, Raucherstatus und Hypertonie. Auch eine Hormontherapie nach der Menopause oder eine orale Kontrazeption hatten keinen Einfluss.

- Kurth Tet al. Migraine and risk of cardiovascular disease in women: prospective cohort study. BMJ. 2016;353:i2610

\section{KOMMENTAR}

Diese große Studie betrachtet nicht wie frühere Arbeiten - nur Schlaganfälle, sondern alle kardiovaskulären Erkrankungen. Das Risiko dafür ist bei Frauen mit Migräne um 40-70\% erhöht. Das ist im ersten Moment erschreckend, es muss allerdings in Relation gesetzt werden. Insgesamt waren die vaskulären Ereignisse sehr selten, sodass die absolute Risikoerhöhung gering ist. Dessen ungeachtet sollten bei Frauen mit Migräne Risikofaktoren abgefragt werden. Im Gespräch ist es wichtig, auf die konsequente Behandlung dieser Faktoren einzugehen. Die Daten sollten jedoch nicht dazu herhalten, Patientinnen mit Migräne Angst vor Schlaganfall und Herzinfarkt zu machen.

Prof. Dr. med. H.-C. Diener

\section{Wie kommen die Mehlkäfer in den Verdauungstrakt?}

Im Rahmen einer Screening-Koloskopie fanden sich bei einer 50-jährigen Mexikanerin im gesamten Kolon dunkle Kügelchen (Abb. A). Bei näherer Inspektion erkannte man offenbar leblose Insekten im Bereich von Zökum (Abb. B) und Colon ascendens (Abb. C). Darauf angesprochen erwähnte die Patientin, dass sie regelmäßig landestypische "gorgojos chinos" zu sich nehme - Rüsselkäfer aus der Überfamilie der Curculionoidea, die im Land unter dem Namen Mehlkäfer bekannt sind. Diese Praxis hat Tradition in der hispanischen Bevölkerung, da man glaubt, dass die im Magen abgestorbenen Käfer ein Enzym absondern, das die Abwehrkräfte des Körpers gegen verschiedene Krankheiten stärken würde - u.a. gegen AIDS und Diabetes. In Online-Foren werden entsprechende Informationen verbreitet; wissenschaftliche Daten hierzu gibt es indes kaum.

Prof. Dr. med. H. S. FüeßI

- Khosla M, Sachdev M, Patel K. Gorgojos Chinos (flour beetle): incidental finding during colonoscopy. Am J Gastroenterol. 2016;111:1514
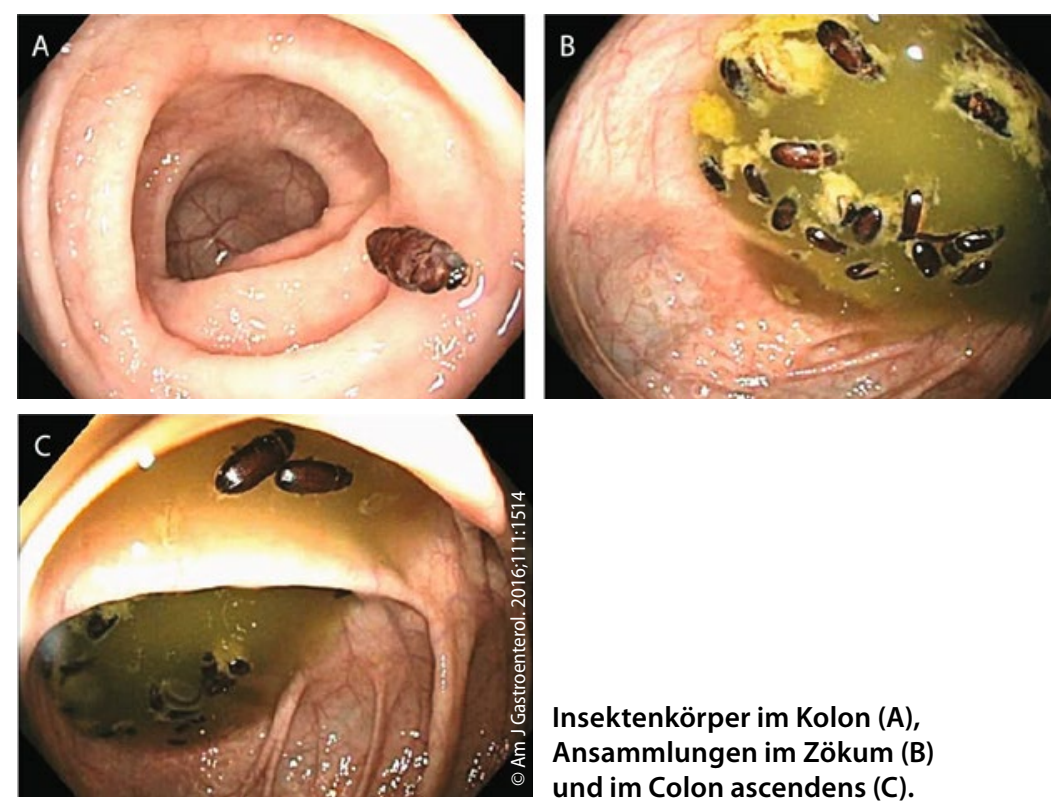

Insektenkörper im Kolon (A), Ansammlungen im Zökum (B) und im Colon ascendens (C). 